Bokanmeldelse - Book Review

Assessment of Responsible Innovation: Methods and Practices, edited by Emad Yaghmaei and Ibo van de Poel, London: Routledge, 2020. 394 pages https://doi.org/10.4324/9780429298998

\title{
Assessing RRI
}

DOI: http://dx.doi.org/10.5324/eip.v15i1.4011

This is an open access article distributed under the terms of the Creative Commons
Attribution 4.0 International License, which permits unrestricted use, distribution, and
reproduction in any medium, provided the original author and source are credited.

Responsible research and innovation (RRI) was implemented as a work package in the EU's Horizon 2020 framework programme for research and innovation, serving the objective of "science with and for society". However, RRI is not part of the current Horizon Europe framework programme. In this context, we might ask what the future holds for RRI and to what extent experiences with RRI over the years can be of use going forward. The book Assessment of Responsible Innovation: Methods and Practices brings together RRI scholars to reflect on how RRI can be practised and assessed in the future.

The book's editors are Emad Yaghmaei and Ibo van de Poel, both working at the University of Delft. In the introduction, they write that the concept of RRI "is still to a limited extent applied in practice" (p. 2). The practical objective of the book is to help stakeholders in matters of monitoring, measuring and managing responsible innovation (p. 2). The book's 14 chapters are organized in three parts: "Reflections on Responsible Innovation", "RRI in companies" and "Responsible Innovation Assessment." Between each of the eight chapters that make up the third part are short descriptions of different RRI best practices.

In the first chapter of the book, philosopher Robert Gianni evaluates the scientific and democratic relevance of RRI for the dialogue between science and society (p. 11). He takes on the difficulties of arriving at a common understanding of the meaning and implication of responsibility, and a homogenous approach to RRI (p. 11). Gianni finds that the principles of freedom, equality and solidarity are shared by all scholars of democracy (pp. 15-16). The concept of responsibility, however, has become increasingly complex, with the rise of technology, and the way in which actors are embedded in systems that often give limited freedom to act (p. 23). Gianni argues for a multi-level understanding of responsibility as responsive to societal challenges and regulating different perspectives (pp. 23-24). Based on his understanding of democracy and responsibility, Gianni gives support to the European Commission (EC)'s six keys of RRI (ethics, science education, gender equality, open access, governance and public engagement), because he finds that they enact a conception of democracy where subjects determine their ends based on moral and epistemic pluralism (p. 31).

In the second chapter, Roger Strand and Jack Spaapen, discuss the creation of an Expert Group on Policy Indicators for Responsible Research and Innovation (p. 42). They were involved in the work to create the expert group and are therefore 
able to provide an inside look at the interplay between ideals and reality in the shaping of RRI in the EU context. They discuss how von Schomberg introduced and defined RRI in 2011. While von Schomberg based RRI on the Collingridge dilemma and constructivist philosophies, the European Commission's adoption of RRI in 2012 reflected neither aspect (pp. 44-45), and the result "was quite far from von Schomberg's vision of philosophically informed self-governance among researchers who sought reflection and deliberation in civil society" (p. 46). Strand and Spaapen argue that governance must acknowledge that there is no predetermined trajectory, nor a "captain" of the ship. There are instead networks with different visions of where we should be headed.

The two chapters that make up the first part of the book complement each other well. Gianni's chapter provides philosophical discussions on the meaning of two key ideals underlying any conception of RRI, democracy, and responsibility, and in my view, such theoretical excursions are necessary as a higher order of RRI selfreflexivity, which goes beyond any concrete project. Without conceptual clarifications of the sort Gianni engages in, RRI risks becoming a fragmented notion, unable to give direction or unity in its application. But just as important as conceptual clarifications are the stories, such as the one provided by Strand and Spaapen, which shed light on what happens when lofty philosophical ideals are institutionalized. After having read these chapters I am left with the impression of an inherent tension in RRI's call for self-governance and self-reflection concerning responsibility. On the one hand, the whole notion of being responsible rests on the assumption that agents (persons or institutions) can "take responsibility" autonomously. On the other hand, RRI is a tool of research and innovation governance which mandates that certain groups, often scientists, become more responsible. If not reflected upon, the tension can result in unrealistic expectations of what governance can achieve and disappointment in people's inability to achieve substantial consensus. The first two chapters in Assessment of Responsible Innovation do not provide solutions to this problem, but they are important contributions to the RRI community's self-reflection on its own ideals and practices.

The second part of the book consists of four chapters, which in different ways discuss how RRI is applied in companies. In her chapter, Agata Gurzawska brings together RRI and Corporate Social Responsibility (CSR) (p. 63). Gurzawska explains that while RRI is largely a top-down approach focusing on ethics and social impact, CSR works from the bottom-up and "concentrates on the impact on community and environment" (p. 73). Gurzawska proposes a new approach of "strategic responsible innovation management" (StRIM). She argues that the approach can incorporate market and non-market stakeholders in a way that creates value and leads to competitive advantage (p. 82), by redefining successful innovation to imply sustainable outcomes (p. 88).

The chapter "Supporting RRI uptake in industry", by Porcari et al. also addresses RRI and CSR. The authors propose a model that aims to "help companies identify RRI implementation strategies during product development - connecting goals, actions and impacts - and a simple methodology to perform qualitative evaluations of its impacts (benefits, barriers and costs)" (p. 118). The authors base their discussion on the PRISMA project, which was dedicated to explore and promote RRI in industry (p. 118). Experiences from the project showed that RRI 
must be implemented at early stages and be considered as a medium- to long-term investment to help companies improve the societal impacts of their disruptive technologies (p. 139).

Cristina Covello and Konstantinos Iatridis investigate the challenges and drivers of oodpreneurial SMEs ("small and medium sized entreprises") when they implement RI (p. 99). Based on a study where they interviewed 19 foodpreneurs, Covello and Iatridis find that foodreprenurial SMEs address many aspects of RI, even if they are unaware of the term 'RI' (p. 111). But the foodpreneurs had problems defining what societal challenges they were driven by. Covello and Iatridis argue that if enterprises do not engage in RI deliberately, they will not be able to ensure that their innovations fulfill societal needs (p. 111). Furthermore, there is a potential conflict between the individualism behind business practices driven by personal values and motivations, and the idea of being responsible towards stakeholders (p. 113).

The final chapter of part two is based on a case study of the European food industry to find out how front-of-pack (FoP) labels "based on voluntary product standards support RRI implementation and reporting in industry" (p. 146). The authors, Inigo et al., acknowledge that the design of products and technologies reflect societal values and that democratic processes are necessary to identify such values (p. 145). But there are several challenges in achieving this, and voluntary standards have been suggested as one way to make RRI more compatible with commercial innovation (p. 146). The authors argue that voluntary standards should be governed by multi-stakeholder initiative or agreement to gain legitimacy ( $p$. 151).

The four chapters that make up the second part of Assessment of Responsible Innovation give insights into how the concept of RRI and its ideals can be implemented beyond national and EU institutions. The increasing importance for private enterprises to embody societal values creates opportunities for doing RRI in new ways. At its best, RRI can be a driver for turning good intentions - or good marketing ploys - into actual commitments to societal values. The biggest challenge for RRI in industry seems to be for companies and entrepreneurs to be willing to enter into dialogue with stakeholders through reflection and deliberation. As Novello and Iatridis aptly point out, even when driven by non-profit motives, the individualism that often accompanies entrepreneurship can be a hindrance to successfully contributing to societal values in a meaningful way.

While the first part of Assessment of Responsible Innovation brought out issues connected to RRI in the public governance context, the second part does so in relation to the private sector. One issue is that, outside of a governance framework that is primarily geared towards societal values, RRI risks being just a call for becoming more responsible. As Gurzawska points out, RRI is a top-down concept. But, as I see it, unless governments demand that private enterprises engage in RRI activities, there is no authority to sanction the top-down structure. In a sense, the chapter on FoP labels offers a solution to this issue, with its discussion of how legitimacy can be gained through voluntary standards set by multi-stakeholder agreements. This kind of solution can provide companies with an authority, which they themselves take part in and which can ensure unity across RRI engagements.

The topic of the first chapter of the third part is the MoRRI (Monitoring the Evolution and Benefits of Responsible Research and Innovation) project, which was 
an effort to develop, implement and assess an RRI monitoring system for the European research area (p. 172). Authors Ingeborg Meijer and Wouter van de Klippe describe how MoRRI translated the EC's six keys into more than 36 indicators meant to capture different aspects of RRI (pp. 179-180). Meijer and van de Klippe find that European RRI has great diversity, but note that further monitoring of RRI should put more emphasis on the institutional level. A monitoring system that focuses on institutional change can provide individuals with the intellectual resources to be able to articulate their values (p. 191).

Tharani et al. start their chapter by discussing how companies, on the one hand, have to continually learn and take in new information in order to gain organizational knowledge and to innovate. On the other hand, the current speed of innovation and lack of trust in institutions lead companies to have to continually update their knowledge in order to anticipate impacts, respond to stakeholder concerns and adapt to the business environment (p. 198). The authors introduce the self-assessment tool COMPASS, which aims to translate RRI into corporate practices and policies (p. 199). By answering a set of questions, an organization can check the policies it has implemented and be guided towards policies for implementing responsible innovation.

Klaassen et al. discuss reflexive monitoring in action (RMA) as a way to deal with the complexity of RRI. RMA is geared towards the difficulties of monitoring and assessing how RRI influences the research and innovation system, and the actors involved in it (p. 223). RMA encourages collective learning practices that can contribute to identifying and overcoming societal barriers to system innovation ( $\mathrm{p}$. 231). It does so through continual reflection on the institutional and societal context of a project, with the help of an appointed reflexive (pp. 232-233). The authors conclude that researchers and innovators will learn to act more responsibly if they engage in reflecting on the meaning of responsibility in their specific context (p. 240).

Mika Nieminen and Veikko Ikonen want RRI evaluation frameworks to pay more attention to contextual embeddedness and organizational factors (p. 248). They describe a practical evaluation approach to embed responsibility thinking into innovation platforms, and for integrating RRI into innovation activity (p. 249). Nieminen and Ikonen discuss the increasing complexity of innovation, involving interaction among several actors and contextual factors. Systemic innovations that require changes in existing technology systems and markets make it necessary to leave behind linear and causal thinking in evaluation, and instead adopt a systems view (pp. 251-252, 255). Nieminen and Ikonen propose a future-oriented evaluation model in the form of a continuous learning loop that involves planning, anticipation, action, and impacts (pp. 256-257). The model emphasizes inclusiveness in the process of developing social-technical innovations, and that different actors should work together to create shared visions (p. 257).

Claudia Werker introduces the concept of RRI systems, and how such systems can be assessed through five steps: 1) identify all stakeholders and their values; 2) develop shared values; 3 ) assess processes and outcomes of the RRI system based on shared values; 4) identify drivers and bottlenecks; 5 ) use shared values to choose solutions to problems (p. 281). Werker points to big data analytics and IoT platforms as potentially having a great influence on assessments of RRI systems in the digital age (p. 283). Big data can help in creating indicators for opportunities 
and challenges in RRI systems (p. 285). IoT platforms can be used to provide information on how persons and organizations involved in RRI processes communicate. This information can then be used to facilitate communication and collaboration which in the end helps in the development of shared values (p. 287).

De Heaver et al. propose a method for assessing responsible innovation in ICT projects. The method is called RRI intensity level (RIL) and evaluates RRI activities according to the extent to which they are meaningful, useful, sensible and effective (p. 298). The authors propose RIL as a tool to help decide on appropriate implementation of RRI in concrete cases (p. 302). With RIL, innovations are to be evaluated along two axes: technology readiness level (TRL) and innovation potential (pp. 305-306). These evaluations are meant to take place at an early stage of a project and assist in selecting the correct type and amount of RRI to implement (p. 303).

In the chapter "The responsible side of innovation," Verburg et al. discuss how RRI can be applied as business practices in organizations. They provide a measurement scale for assessing RRI in contexts where innovations are likely to occur (p. 319). The authors have developed their measurement instrument by bringing together RRI and literature on moral creativity. They find that RRI neglects the role of employees in driving innovation in business contexts (pp. 325326). Hence, the measurement scale that is being proposed is geared towards the level of employees in businesses. The authors emphasize that responsibility should not be separated from and come after the creation and application of innovation (p. 329). Focusing on the ethical appropriateness of novel ideas, the aim is to make responsibility a part of the entire innovation process.

In the final chapter, Ibo van de Poel aims to "highlight some of the potential pitfalls of RRI measurement, assessment and incentivizing" (p. 340). He finds that there are three reasons making it difficult to measure RRI: 1) lack of consensus on the definition of RRI (p. 350); 2) that RRI interventions do not aim at an absolute level of responsibility, but are based on a societal point of view (p. 351) and 3) that RRI is a normative notion (p. 351). van de Poel suggests that three different rationales for RRI assessment can be distinguished: learning, accountability, and incentivizing (p. 355). Tensions exist between all of these rationales, which makes them hard to combine (p. 356), and if people are not clear about the rationales or try to combine them, they risk ending up with inappropriate assessment methods (p. 356).

Several of the different approaches to assessing responsible innovation discussed in this book emphasize the importance of not only focusing on outcomes, but on the process of innovation itself, and the actors and institutions involved. Systems approaches to innovation have existed since the 1980s (Schot \& Steinmueller, 2018). Now, RRI scholars and practitioners are working towards clarifying what it means to take a systems approach to responsibility in RRI activities and assessments as well. But is it possible to fully adopt systems approaches without overstepping the boundaries of RRI? Both von Schomberg's foundational definition of RRI and the four dimensions in the RRI framework of Stilgoe et al. are oriented towards innovations driven forward by scientific research. This implies that even though Schomberg and Stilgoe et al. promote deliberation and reflection among a wide variety of stakeholders, their approaches to a certain extent imply a linear model of innovation, as well as a systems approach. If RRI is 
to fully take on the systems approach, non-research institutions and actors will have to be seen as at least equally important in creating innovation as those engaging in scientific research.

RRI scholars will have to figure out whether they want to hold on to a scienceoriented RRI, or fully embrace the systems approach. I commend the way in which several of the authors of this volume have broadened the scope of RRI towards a genuinely systems-oriented approach. But there is also something to be said for the approaches that are more in line with a linear approach, such as the COMPASS tool and RIL. These approaches are suitable for contexts where it is possible to envisage a more or less step-wise innovation trajectory that can be assessed either at an early stage or throughout the process. It might be true today that many, if not most, innovations are more or less systemic in nature. But in those cases where innovation processes are driven forward by research and development, there will still be a need for methods that focus on stakeholder involvement and foresight geared towards tackling the fundamental problem of the Collingridge dilemma.

Assessment of Responsible Innovation does more than present the interested reader with several time-tested ways of conducting and assessing RRI. It offers insights into the many opportunities and challenges that face anyone who tries to do research and innovation responsibly. The book shows that scholars and practitioners are venturing beyond the path that was first set out when the EC adopted RRI in 2012. This creative exploration is key to be able to utilize the theoretical and empirical insights and the practical know-how that has been accumulated so far. They show that simply trying to act responsibly is not enough; we must continually put our own ethical assumptions, values and visions under scrutiny.

Alexander Myklebust, NTNU

\section{Literature}

Schot, J., \& Steinmueller, W. E. (2018). Three frames for innovation policy: R\&D, systems of innovation and transformative change. Research Policy, 47(9), 15541567. https://doi.org/10.1016/j.respol.2018.08.011 